\title{
Vínculos Empregatícios de Médicos de Família e Comunidade no Brasil, 2004
}

\author{
Geographical Distribution of Employment \\ Contracts of Family and Community \\ Physicians in Brazil in 2004
}

\author{
Maurício de Garcia Bolze \\ Paul Douglas Fisher ${ }^{I}$ \\ Maria Ceci Araujo Misoczky ${ }^{I}$ \\ Ronaldo Bordin ${ }^{I}$
}

\section{PALAVRAS-CHAVE \\ - Epidemiologia. \\ - Gestão em Saúde. \\ - Recursos Humanos em Saúde. \\ - Atenção Primária à Saúde. \\ - Políticas de Saúde.}

\begin{abstract}
Since it was introduced in Brazil in 1994, the Family Health Program (FHP) has been the main strategy for restructuring primary health care in the country. In this context, the work of family physicians in the community has been essential for the program's success. This study describes the geographical distribution of family physicians in Brazil, based on employment contracts listed in the National Registry of Health Institutions, and the relationship to the infant mortality rate, human development index, mean schooling, GDP, and monthly income in 2004. This was an ecological study presenting the variables geographically, by micro-region. The correlation coefficients between number of family physicians and infant mortality rate, human development index, and GDP were, respectively: $0.17,0.18$, and 0.74 . Although nearly all the micro-regions had family physicians, fewer than $12 \%$ had three or more family physicians (as recommended by FHP guidelines). A decade into the Family Health Plan in Brazil, the distribution of family physicians was still uneven and insufficient in most micro-regions.
\end{abstract}




\section{INTRODUÇÃO}

O Sistema Único de Saúde (SUS) é um dos maiores sistemas públicos de saúde do mundo, além de ser um dos únicos a garantir assistência integral e gratuita para a totalidade da população ${ }^{1}$. Apresenta como diretrizes constitucionais a universalidade, integralidade e equidade das ações de saúde; é operacionalizado por um sistema regionalizado, hierarquizado, descentralizado e sob controle social. Seu arcabouço jurídico encontra-se na Constituição Federal de 1988 e na Lei Orgânica de Saúde de 1990. Sua estruturação alterou o modelo de atenção até então vigente, possibilitando priorizar as ações de promoção e prevenção, mantendo as ações curativas e de recuperação.

Passadas quase duas décadas de sua criação, o SUS orientou grandes movimentos relativos ao trabalho em saúde. Inicialmente deu-se o deslocamento das funções executoras para o âmbito municipal, cabendo às esferas superiores o papel de regulamentação e orientação técnica e financeira, almejando alcançar maior extensão da cobertura dos serviços de saúde. De forma complementar a esse momento, foi necessária uma mudança no perfil requerido aos trabalhadores para viabilizar a execução das diretrizes do SUS de forma plena.

As experiências internacionais — na Inglaterra e Canadá, por exemplo - mostram que, quando o Sistema de Saúde de um país é focado na Atenção Primária em Saúde (APS), se conseguem melhores resultados e maior equidade em saúde, com menores custos e maior satisfação dos usuários². No Brasil, a reorganização da APS se deu por meio da implantação, a partir de 1994, da Estratégia Saúde da Família (ESF)³. A ESF altera as práticas convencionais da assistência, baseando-se na vigilância à saúde, na territorialização/adscrição da clientela, na atenção integral à saúde e no trabalho multiprofissional, quando o médico não é mais o único responsável pelos pacientes ${ }^{4}$.

A implantação das equipes de Saúde da Família no Brasil se deu, prioritariamente, em áreas de risco, segundo o Mapa da Fome do Instituto de Pesquisas Econômicas Aplicadas (Ipea $)^{5}$. Segundo estudos da primeira metade da década ${ }^{6}$, as equipes de Saúde da Família estavam localizadas principalmente nas zonas interioranas e junto às áreas mais pobres, regiões até então praticamente sem acesso aos serviços de saúde. Neste contexto, uma das metas do Ministério da Saúde era ampliar a ESF nas regiões metropolitanas ${ }^{3}$.

A maioria desses estudos tem como unidade de pesquisa a equipe de Saúde da Família ${ }^{7,8}$, sendo poucos os especificamente relacionados ao Médico de Família e Comunidade (MFC) ou generalista, integrante da equipe mínima definida para a Saúde da Família ${ }^{6,9}$.

Considerando como chave o papel desempenhado pelo MFC dentro da política da ESF e a importância da adequada alocação desses profissionais para garantir seu acesso à população ${ }^{2}$, este estudo objetiva descrever a distribuição geográfica dos postos de trabalho em Medicina de Família e Comunidade no Brasil, com o intuito de ser mais um instrumento epidemiológico a auxiliar em futuras tomadas de decisão.

\section{MÉTODO}

Foi realizado um estudo do tipo ecológico, utilizando-se como fonte de coleta de dados o Cadastro Nacional de Estabelecimentos de Saúde - CNES (http:/ / cnes.datasus.gov.br/).

Do CNES foi extraído o número total de vínculos de contratação de médicos por município, independentemente de especialidade ou porte assistencial (UBS, hospital, sistema público ou complementar, etc.) e, destes, especificamente os de Médicos de Família e Comunidade. Na sequência, esses dados foram sistematizados segundo as 558 microrregiões do IBGE, que agregam os 5.561 municípios brasileiros. Esses dados fazem parte do "Estudo para Identificação de Necessidades de Profissionais e Especialidades na Área da Saúde", demandado pela Secretaria de Gestão do Trabalho e Educação na Saúde, do Ministério da Saúde, e realizado junto a cinco universidades brasileiras.

Emprega-se como objeto de pesquisa não o número de médicos ou especialistas em MFC propriamente dito, mas o vínculo de trabalho médico que é a relação contratual com o serviço. Ou seja, um médico pode ter mais de um vínculo de trabalho ou ser contratado sem uma dada especialidade para suprir a carência da mesma, por exemplo.

A partir do site do IBGE foram coletadas as seguintes variáveis: anos de estudo das pessoas maiores de dez anos, Produto Interno Bruto (PIB) e o rendimento nominal mediano mensal do trabalho principal das pessoas com dez anos ou mais ocupadas na semana de referência, além das estimativas populacionais.

Do site do DataSUS foram extraídos a população residente e os números de nascidos vivos e óbitos em menores de um ano por município. O coeficiente de mortalidade infantil foi calculado dividindo-se o número de óbitos pelo número de nascidos vivos e multiplicado por 1.000 .

O Índice de Desenvolvimento Humano (IDH) foi adquirido a partir do site do Programa das Nações Unidas para o Desenvolvimento. (www.pnud.org.br/idh/).

Os dados foram trabalhados no software Microsoft ${ }^{\circledR}$ Excel 2002 SP3. Os gráficos e coeficientes de correlação foram obtidos com o SPSS 12.0 for Windows, e a apresentação gráfica em forma de mapa via Tab para Win32 e Wine versão 3.4.

O presente estudo foi submetido à aprovação do Comitê de Ética da Universidade Federal do Rio Grande do Sul (Processo $\left.n^{\circ} 10.945\right)$. 
TABELA 1

Características das variáveis estudadas, por microrregião

\begin{tabular}{|c|c|c|c|c|}
\hline Variável & Mínimo & Máximo & Média & Desvio-Padrão \\
\hline População residente (em 1.000 habitantes) & 2,2 & $13.232,57$ & 320,93 & 837,5 \\
\hline Vínculos médicos & 1 & 27.341 & 581,44 & $1.701,79$ \\
\hline Densidade de vínculos médicos (por 10 mil habitantes) & 0,55 & 51,89 & 15,35 & 8,12 \\
\hline Vínculos em Medicina de Família e Comunidade (MFC) & 0 & 829 & 39,11 & 60,76 \\
\hline Densidade de vínculos em MFC (por 10 mil habitantes) & 0 & 10,15 & 1,71 & 1,1 \\
\hline \% de vínculos em MFC sobre o total de vínculos médicos & 0 & 100 & 14,28 & 11,41 \\
\hline Coeficiente de mortalidade infantil & 0 & 45,5 & 14,95 & 7,18 \\
\hline PIB (em milhão de reais) & 8,37 & $185.214,39$ & $2.786,88$ & $10.265,97$ \\
\hline PIB (em mil reais) per capita & 0,97 & 97,41 & 6,92 & 7,18 \\
\hline Rendimento mediano mensal (em R\$) & 75,33 & 542,45 & 223,73 & 83,94 \\
\hline Índice de Desenvolvimento Humano (I(IDH) & 0,512 & 0,862 & 0,71 & 0,079 \\
\hline Anos estudados & 1,9 & 7,7 & 4,8 & 1,26 \\
\hline
\end{tabular}

\section{RESULTADOS}

Em 2004, existiam no Brasil 324.444 vínculos de trabalho médico cadastrados no CNES, sendo 21.823 (6,7\%) vínculos em MFC. A Tabela 1 apresenta as variáveis analisadas neste estudo, agregadas segundo microrregiões. Por meio da análise dos valores exibidos pode-se ter uma idéia do quanto irregular é a distribuição da maioria das variáveis.

É relatada a existência de vínculos de trabalho em MFC em $98,7 \%$ das microrregiões do Brasil. Entretanto, eles se concentravam principalmente na Região Nordeste: das 20 micror-

FIGURA 1

Densidade de Vínculos em Medicina de Família e Comunidade (MFC) por Microrregião do Brasil, 2004

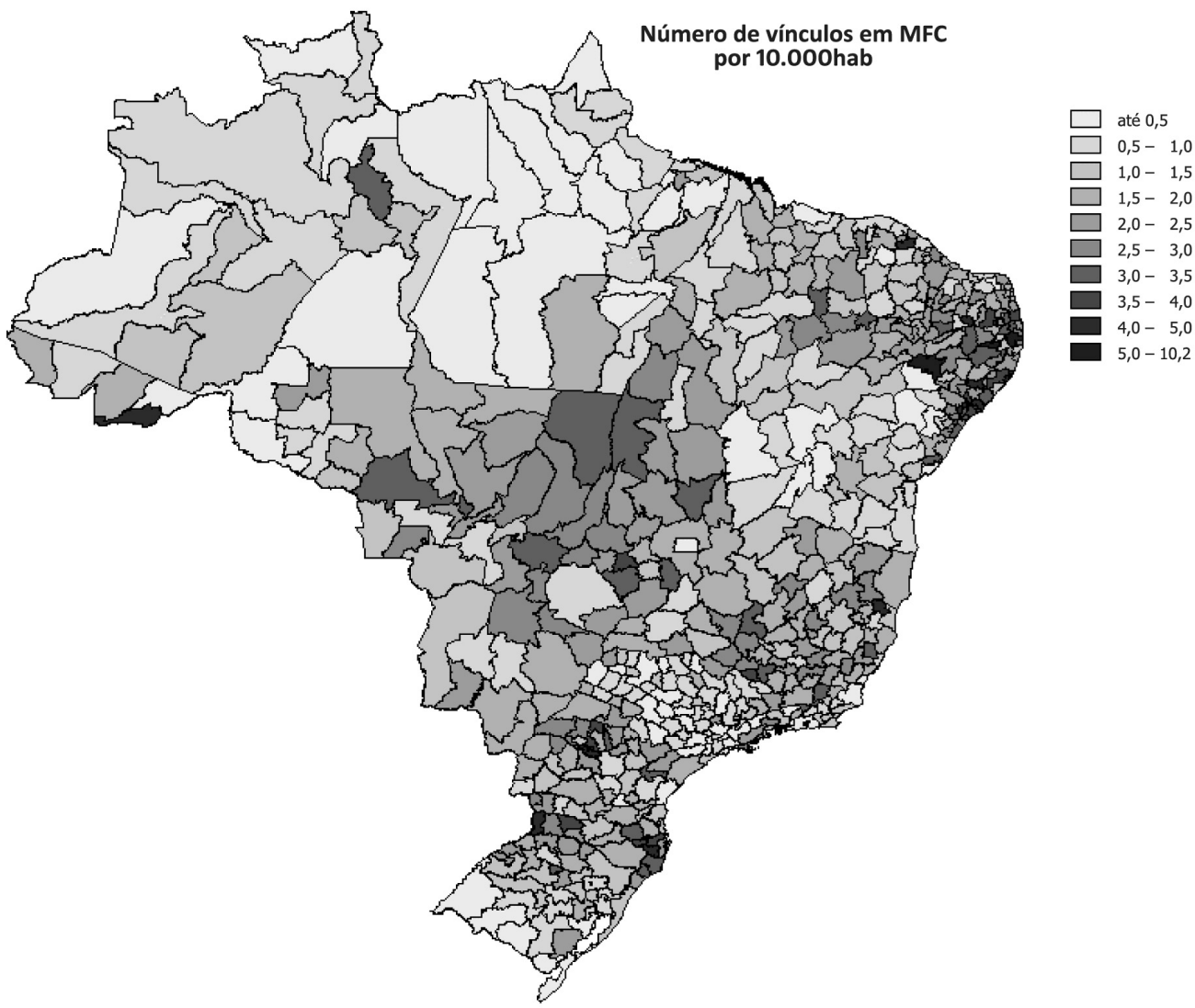

$\left.408\right|_{34(3): 406-411 ; 2010} ^{\text {REVISTA BRASILEIRA DE EDUCAÇ̧̃o MÉDICA }}$ 


\section{GRÁFICO 1}

Densidade acumulada de vínculos em Medicina de Família e Comunidade (MFC) nas microrregiões do Brasil, 2004

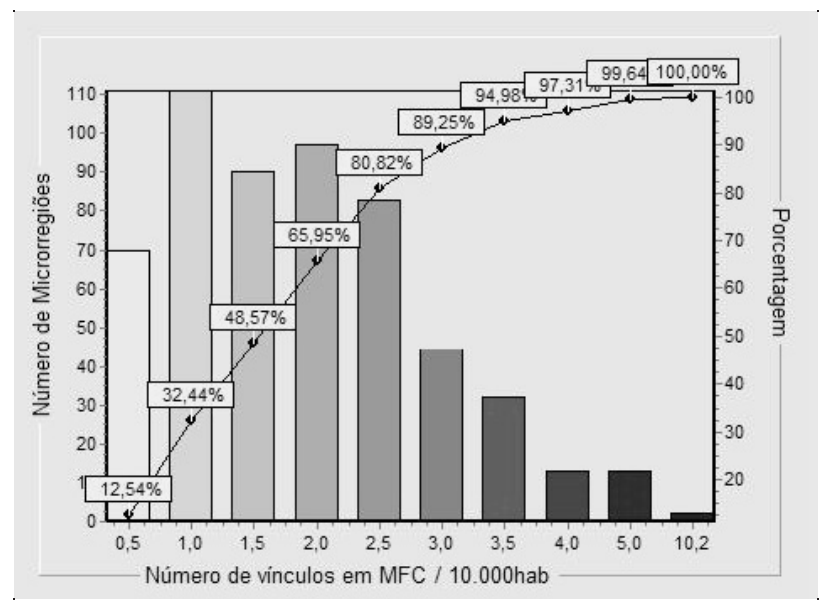

regiões com maior densidade de vínculos por habitante, 13 estão localizadas nessa região.

A distribuição dos vínculos em MFC de acordo com a população de cada microrregião se encontra na Figura 1 e no Gráfico 1, que mostram a distribuição geográfica irregular desses vínculos. As microrregiões com maior densidade de vínculos se acham, principalmente, no Nordeste e no centro do País.

O Gráfico 1 mostra que o número de vínculos de trabalho em MFC ainda era muito pequeno em 2004. Considerando-se que a ESF preconiza uma população de até 3.500 pessoas para cada MFC, menos de $11 \%$ das microrregiões estudadas apresentava três ou mais vínculos para cada 10 mil de seus habitantes.

As análises de correlação entre as variáveis estão representadas na Tabela 2 .

TABELA 2

Coeficientes de Correlação

\begin{tabular}{lc}
\hline Variáveis correlacionadas & r \\
\hline População residente x número total de vínculos médicos & 0,98 \\
Vínculos médicos x vínculos em MFC & 0,83 \\
População residente x vínculos em MFC & 0,79 \\
PIB x vínculos em MFC & 0,74 \\
Anos estudados x vínculos em MFC & 0,28 \\
Renda média x vínculos em MFC & 0,25 \\
IDH x vínculos em MFC & 0,18 \\
Mortalidade infantil x vínculos em MFC & 0,17
\end{tabular}

$r$ : coeficiente de correlação; MFC: Medicina de Família e Comunidade; PIB: produto interno bruto; IDH: índice de desenvolvimento humano.

FigURA 2

Correlação entre o PIB (em R\$ 1.000) e o número de vínculos em Medicina de Família e Comunidade (MFC), 2004

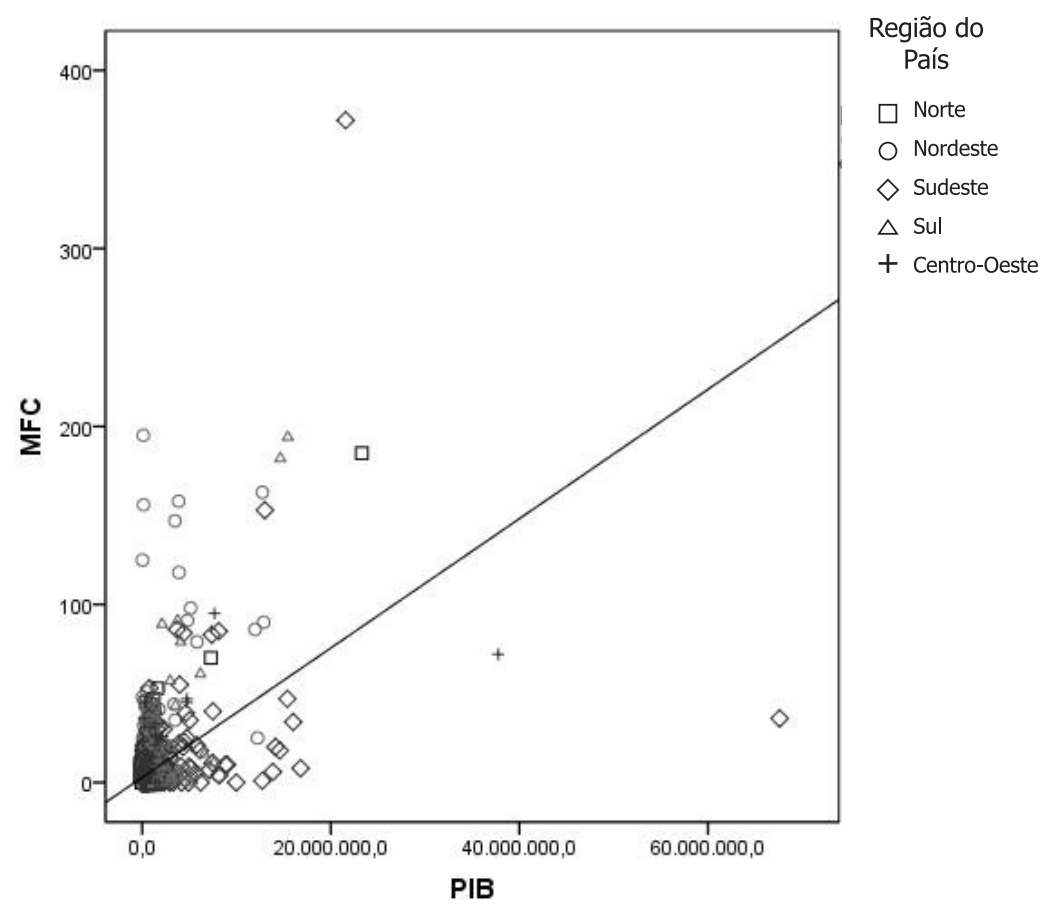


O número de vínculos em MFC apresentou tendência a se correlacionar mais fortemente com o PIB (Figura 2) e com a população residente. Já com os demais indicadores, as correlações não passaram de fracas.

\section{DISCUSSÃO}

Antes da análise dos dados, saliente-se que todos são provenientes de fontes secundárias, não originalmente coletados para este estudo. Foram empregadas bases de dados do DataSUS, do IBGE, do CNES e do PNUD. Enquanto as informações do PNUD e principalmente do DataSUS são facilmente acessadas e exportadas, o mesmo não se aplica às tabelas do IBGE e do CNES. As informações destas bases são bem mais difíceis de encontrar e, uma vez selecionadas, estão em formatos que demandam tabulação mais trabalhosa que as demais.

Saliente-se, também, que as bases do CNES e do DataSUS são alimentadas diretamente pela administração de cada município brasileiro. Considerando a quantidade de informações e a grande variedade de situações administrativas, é necessário ressalvar uma provável incorreção em alguns dados, já que estes não foram conferidos (possível viés de aferição). $\mathrm{O}$ emprego da unidade de análise baseado nas microrregiões do IBGE objetivava amenizar essas grandes variações encontradas entre os municípios. Os grandes desvios-padrão em relação às médias encontrados na população residente e nos números de MFC e médicos totais exemplificam esta situação.

Conforme a ESF, o PSF - e, logo, os vínculos em MFC veio para suprir uma demanda reprimida em saúde, especialmente em pequenos municípios e regiões afastadas dos grandes centros populacionais, onde historicamente se concentrou o maior número de médicos. Essas regiões, portanto, também apresentavam os piores índices de desenvolvimento e os piores indicadores de saúde.

Entretanto, no ano de 2004, a distribuição dos vínculos de trabalho em MFC não seguia muito claramente nenhum dos indicadores estudados, conforme demonstram os fracos coeficientes de correlação encontrados. Indicadores importantes, como a taxa de mortalidade infantil e o índice de desenvolvimento humano, apresentaram apenas pequenas tendências de correlação com o número de vínculos em MFC.

\section{CONCLUSÕES}

Após dez anos de implantação da ESF no Brasil, ainda era bastante irregular a distribuição dos vínculos de trabalho em Medicina de Família e Comunidade em 2004.

Mesmo que os dados extraídos do CNES não reflitam plenamente a realidade, era de se esperar que mostrassem correlações mais fortes pelo menos com alguns dos indicadores sócio-geográficos selecionados para a análise. Apesar de ter sido descrito que a expansão inicial do PSF se deu, a partir de 1998, em regiões mais pobres, menos povoadas e do interior ${ }^{3}$, já em 2004 essa polarização não era mais tão evidente.

Segundo Viana e Dal $\mathrm{Poz}^{5}$, quatro são os principais elementos dificultadores de melhor expansão do PSF no Brasil: a própria estrutura do Ministério da Saúde, a lenta formação de recursos humanos, as resistências corporativas (Conselhos e Associações de Medicina e Enfermagem) e a noção de que atenção primária é sinônimo de tecnologia simplificada.

Os crescentes incentivos dos gestores em saúde descritos por Marques e Mendes ${ }^{10}$ ainda não podiam ter reflexos percebidos em 2004, pois na maioria das microrregiões brasileiras a densidade de vínculos ainda estava abaixo do considerável como suficiente. A estrutura pouco flexível e lenta do Ministério da Saúde dificulta a descentralização. Por outro lado, ações como o Pacto pela Saúde e a Política Nacional de Atenção Básica demonstram que não foi esquecida a necessidade de revisão e adequação das normas regulamentadoras da APS ${ }^{11}$.

Outro fator que pode estar influenciando a distribuição irregular dos vínculos em MFC é a carência de especialistas. Segundo dados do Sistema de Informação em Atenção Básica (Siab), em maio de 2007 já existiam mais de 27 mil EqSF, enquanto o número de médicos com formação em Saúde da Família não chega hoje a 2.800 (www.mec.gov.br). Isto significa que a maioria dos médicos que trabalham como MFC na verdade só exercem tal função, sem formação específica. A dificuldade de encontrar profissionais capacitados para ocupar os postos de trabalho abertos poderia alocá-los nos grandes centros urbanos ou municípios capitalizados, com poder de oferecer melhores salários.

Apesar de não ser o foco principal de nosso estudo, é importante destacar os resultados evidenciados por Starfield et $a .^{2}$, que encontrou vários trabalhos que correlacionam maiores densidades populacionais de médicos generalistas com melhores indicadores de saúde nas mais variadas áreas da saúde pública.

A continuidade dos estudos em relação ao trabalho em saúde poderá evidenciar mais claramente de que modo vem acontecendo a distribuição dos vínculos em MFC e dos próprios EqSF no Brasil. Uma análise da evolução do número desses ao longo dos anos serviria para orientar e otimizar a alocação de incentivos para a criação de novas equipes.

\section{REFERÊNCIAS}

1. Souza RR. O Sistema Público de Saúde Brasileiro. Seminário Internacional Tendências e Desafios dos Sistemas de 
Saúde nas Américas; 11 a 14 ago. 2002; São Paulo, SP. Brasília: Ministério da Saúde; 2002.

2. Starfield B, Shi L, Macinko J. Contribution of Primary Care to Health Systems and Health. Milbank Q. 2005;83(3):457502.

3. Ministério da Saúde. Secretaria de Gestão Participativa. Saúde da Família: panorama, avaliação e desafios. $2^{\circ}$ Seminário de Gestão Participativa: Fórum de Conselhos Municipais de Saúde da Região Metropolitana I; 2004; Rio de Janeiro, RJ. Brasília: Ministério da Saúde; 2005. (Série D. Reuniões e Conferências, Série Cadernos Metropolitanos).

4. Ministério da Saúde. Programa Saúde da Família. O programa saúde da família e a atenção básica no Brasil. Brasília: Ministério da Saúde; 2002.

5. Viana ALD, Dal Poz MR. A Reforma do Sistema de Saúde no Brasil e o Programa de Saúde da Família. Physis. 2005;15(Supl.):225-64.

6. Ministério da Saúde. Secretaria de Atenção à Saúde. Departamento de Atenção Básica. Avaliação normativa do Programa Saúde da Família no Brasil: monitoramento da implantação e funcionamento das equipes de saúde da família: 2001-2002. Brasília: Ministério da Saúde; 2004. (Série C. Projetos, Programas e Relatórios).

7. Ministério da Saúde. Secretaria de Atenção à Saúde. Departamento de Atenção Básica. Coordenação de Acompanhamento e Avaliação da Atenção Básica. Dez Anos de Saúde da Família no Brasil. Informe da Atenção Básica. 2004;5(21):1-2.

8. São Paulo. Prefeitura Municipal. Secretaria Municipal de Saúde. Implantando o Programa de Saúde da Família no Município de São Paulo: Balanço dos 20 meses. São Paulo: Programa Saúde da Família; 2002.
9. Machado MH, Pereira S. Los recursos humanos y el sistema de salud en Brasil. Gac Sanit. 2002;16(1):89-93.

10. Marques RM, Mendes A. Atenção Básica e Programa de Saúde da Família (PSF): novos rumos para a política de saúde e seu financiamento? Ciênc Saude Coletiva. 2003;8(2):403-15.

11. Ministério da Saúde. Secretaria de Atenção à Saúde. Departamento de Atenção Básica. Política nacional de atenção básica. Brasília: Ministério da Saúde; 2006. (Série A. Normas e Manuais Técnicos, Série Pactos pela Saúde 2006, v. 4).

\section{CONTRIBUIÇÃO DOS AUTORES}

Maurício de Garcia Bolze e Paul Douglas Fisher participaram do desenho do estudo, aquisição, análise e interpretação de dados e da redação do artigo, da versão inicial à encaminhada para publicação. Maria Ceci Araujo Misoczky e Ronaldo Bordin participaram da concepção e desenho do estudo, aquisição, análise e interpretação de dados e da redação do artigo, da versão inicial à encaminhada para publicação.

\section{CONFLITO DE INTERESSES}

Declarou não haver.

\section{ENDEREÇO PARA CORRESPONDÊNCIA}

Ronaldo Bordin

Programa de Pós-Graduação em Epidemiologia, Faculdade de Medicina - UFRGS

Rua Ramiro Barcelos, 2600 — sala 428

Santana - Porto Alegre

CEP 90035-003 RS

E-mail: ronaldo.bordin@ufrgs.br 